\title{
Sarcomatoid carcinoma of the tongue: a rare malignancy in an unusual site -a case report with uncommon history
}

\begin{abstract}
Sarcomatoid (Spindle cell) carcinoma is an unusual variant of squamous cell carcinoma. It is a biphasic tumour composed of both elements sarcomatous and carcinomatous. The mean age at presentation was 58 years with a range of $14-87$ years. In index case had not history of predisposing factors like smoking, tobacco chewing, alcohol consumption and previously irradiation of head neck region patient, despite has highly aggressive tumor at unusual site. That led us to write this case report. We should search many other factors that causes for this tumor than common factors. So, we were described that case to identified the different causes of the tumor, better understanding and awareness of this rare malignancy.
\end{abstract}

Keywords: sarcomatoid carcinoma, tongue, genetic factors
Volume 10 Issue 2 - 2018

\author{
Khairwa $A^{1}, 2$ \\ 'Department of Pathology, ESIC Model Hospiatal, India \\ ${ }^{2}$ Department of Pathology, Holy Family Hospital, India
}

Correspondence: Anju Khairwa, Department of Pathology, ESIC Model Hospiatal, Holy Family hospital, FTA, 504, AV Nagar, New Delhi, India,Tel +9198I0436/38,

Emailanjukhairwa@gmail.com

Received: May 09, 2017 | Published: April II, 2018

\section{Introduction}

Sarcomatoid (Spindle cell) carcinoma is an unusual variant of squamous cell carcinoma. It also called pseudosarcoma, collision tumor and pseudosarcomatous carcinoma. ${ }^{1}$ It is a biphasic tumour composed of both elements sarcomatous and carcinomatous. The mean age at presentation was 58 years with a range of 14-87 years. Male more commonly involved than female. This is more common in the larynx than in the oral cavity and oropharynx. It is rarely arise from tounge. Predisposing factors are smoking, alcohol consumption and previously irradiation of head neck region. Sarcomatoid carcinoma is a poorly differentiated variant of squamous cell carcinoma. ${ }^{2}$ It is have an aggressive behavior. ${ }^{3}$ Despite several immunohistochemical, electron microscopic, and genetic studies, precise histogenesis of it is quite controversial. In index case uncommon history of patient despite has highly aggressive tumor at unusual site, which lead us to write this case report. We should searched many other factors that causes for this tumor than common causes pre-existing SCC or radiation therapy, smoking, alcohol and any tobacco. So, we were described that case to identified the different causes of the tumor, better understanding and awareness of this rare malignancy

\section{Case report}

A 44-year-old man was admitted to the department of ENT with a painless polypoid lesion located in the dorsal lateral aspect of the tongue. Clinical history revealed that he had a non-healing lesion in the right lateral border of the tongue 1year back and it was increased in size rapidly. He had difficulty in eating, swallowing and specking. The patient did not have a history of pre-existing SCC or radiation therapy, smoking, alcohol and any tobacco. On physical examination, a polypoid, exophytic mass was observed which were $2.2 \times 2 \times .8 \mathrm{~cm}$ in size and of firm, rubbery, and nontender character. The patient underwent operation; polypoid mass was resected with wide surgical margins and in combination with right extended supraomohyoid neck dissection. The lesion was operated and partial glossectomy specimen done and an excision biopsy was sent in our department for histopathological examination and frozen for margins. Frozen sections of all margins are free of tumor. Grossly the mass is polypoid arising from dorsolateral aspect of tongue. Hemi-glossectomy specimen measuring $3 \times 2 \times 1 \mathrm{~cm}$. The polypoid growth measuring $2.2 \times 2 \times .8 \mathrm{~cm}$ and with focal ulceration. The cut surfaces of resected specimen had heterogeneous appearance and were firm, whitish and hemorrhagic areas. On cutting Right extended supraomohyoid neck dissection was illustrated 12 lymph nodes. Microscopic examination revealed a biphasic tumor composed of malignant epithelial component and malignant spindle cell type sarcomatous stroma. The epithelial component was showing round to oval nuclei, moderate nuclear pleomorphism, coarse nuclear chromatin, prominent nucleoli and moderate amount of eosinophilic cytoplasm with focal keratinization. The mesenchymal component revealed intersecting and interlacing bundles or fascicles of spindle shaped cells, having elongated nuclei with marked pleomorphism, inconspicuous nucleoli and scant to moderate amount of cytoplasm. In addition frequent mitotic figures and focal areas of necrosis also noted. The surface lining epithelium is ulcerated and acutely inflamed. All resected margins, omohyoid muscle, salivary gland and all 12 lymph nodes are free of tumor. Thus we diagnosed this case as spindle cell carcinoma (Sarcomatoid carcinoma) of the tongue according to the WHO 2005 classification, in which the diagnostic criteria were defined as the presence of malignant spindle cells in addition to the demonstration of invasive or in situ squamous cell carcinoma, or any evidence of epithelial differentiation of spindle cells. ${ }^{4}$

\section{Discussion}

Sarcomatoid carcinoma is rare variant of squamous cell carcinoma of tongue. It has both malignant epithelia cells and malignant spindle cells. The diagnosis can be very difficult, especially when SCC component is not obvious. The spindle cell component may resemble many lesions, ranging from benign reactive ones like radiation-induced granulation tissue to malignant lesions like fibrosarcoma. Therefore, to establish a correct diagnosis, any clue of epithelial component should be carefully sought in suspected lesions. ${ }^{4}$ There are different theories have been proposed to explain histogenetic nature of spindle cells. According to these theories is that spindle cells and epithelial 
cells are arising simultaneously from separate stem cells, the nature of the spindle cell component as an atypical reactive proliferation of the stroma and cells of both spindle and epithelial components have the same monoclonal origin, and "dedifferentiation "or "transformation" to spindle cells has been occurred. ${ }^{5}$ Histochemical and ultrastructural studies showed that spindle cells presented same characteristics with epithelial cells. ${ }^{6}$

Most of the studies reported Sarcomatoid carcinoma, like squamous cell carcinoma, affects primarily men in 6-7th decades and is strongly associated with smoking and alcohol consumption. ${ }^{4}$ Our case is unique than other cases because he below than mean age but age was within typical range and he had not history of any tobacco, alcohol and no any viral/bacterial infection. Oktay et al., ${ }^{1}$ reported a case of spindle cell carcinoma of tongue which had history of smoking and alcohol consumption with recurrent lesion. Similar to our case Oktay et al., ${ }^{1}$ did not reported any positive lymph node but they observed mandible was invaded by the tumor. In head and neck region, the most common localizations of spindle cell carcinoma are larynx, oral cavity, skin, tonsil, and pharynx. ${ }^{5}$ In our case, the spindle cell carcinoma had arisen from the tongue which is one of the rare sites.

These tumors usually grow up rapidly that is seen in our case. Histologically, mesenchymal component typically forms the bulk of the tumor and epithelial component often blends into it. ${ }^{2}$ Malignant pleomorphic cells may exhibit hypo and hyper-cellular areas and sometimes storiform pattern. Typical and atypical mitotic Figure 1 is usually prominent. Giant cells may also be found. Our case showed the characteristic microscopic features as above mencentioned including but presence of giant cells not seen. Few studies observed osteosarcomatous, chondrosarcomatous, or rhabdomyosarcomatous differentiation may be present in especially previously irradiated patients. ${ }^{4,7}$ We did not see such areas in our index case. Regional lymph node metastasis is reported up to $25 \%$ of cases and distant metastasis is less common. ${ }^{4}$ In our case all lymph node are free of tumor. Biradar et al., ${ }^{8}$ reported spindle cell carcinoma of tongue which had history of tobacco use, poor oral hygiene, alcohol abuse, and previous ionizing irradiation of the area and no lymph node involment. In the present case report, the patient had no similar history.

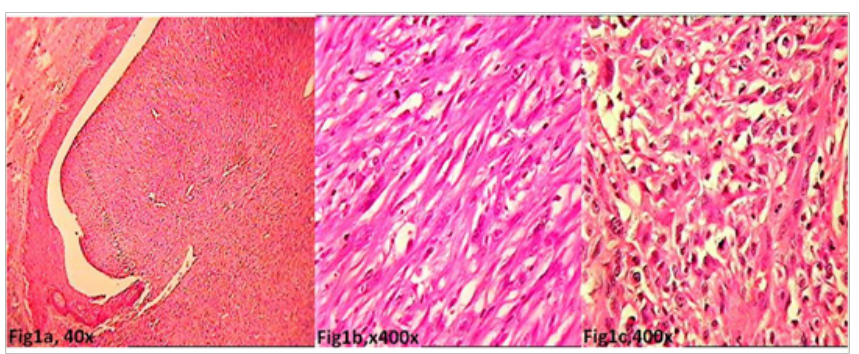

Figure I A panel of microphotographs of Sarcomatoid carcinoma, (A) a polypoid mass arising from tongue (40X, H\&E stained). (B) mesenchymal component comprising intersecting and interlacing bundles or fascicles of spindle shaped cells, having elongated nuclei with marked pleomorphism, inconspicuous nucleoli and scant to moderate amount of cytoplasm (400X, H\&E stained). (C) Epithelial component has round to oval nuclei, moderate nuclear pleomorphism, coarse nuclear chromatin, prominent nucleoli and moderate amount of eosinophilic cytoplasm with focal keratinization (400X, H\&E stained).

There are most of studies shown etiology of spindle cell carcinoma of oral cavity are tobacco use, poor oral hygiene, alcohol abuse, and previous ionizing irradiation. But these factors were not associated with our case. So, there may be any genetic factor or unknown etiological factors were also associated. Spindle cell carcinomas should be treated like the squamous cell carcinomas in the same stage. Thompson et al., ${ }^{9}$ reported that surgically treated patients had a better prognosis than the patients treated with radiotherapy alone. In the oral region, surgery is accepted as the best treatment choice. The role of chemotherapy in the treatment of Sarcomatoid carcinoma is not explained.

\section{Conclusion}

In the conclusion, we present a rare case of Sarcomatoid carcinoma of tongue with unusual history. Based on the limited number of studies in literature, its exact pathogenesis, clinical behavior, and long term prognosis have not been well understood yet. The etiology of spindle cell carcinoma of oral cavity not cleared yet. So, large scale studies and genetic association should be rule out. Despite its challenging character, clinical history and presence of squamous cell component or determination of epithelial nature of spindle cells would be guide the pathologist to diagnosis such cases. In addition a differential diagnosis of spindle cell tumor of the head and neck region should always be mentioned if malignant spindle cells are seen.

\section{Acknowledgment}

None.

\section{Conflict of interest}

None.

\section{References}

1. Oktay M, Kokenek-Unal TD, Ocal B, et al. Spindle Cell Carcinoma of the Tongue: A Rare Tumor in an Unusual Location. Patholog Res Int. 2011;2011:572381.

2. Stelowand EB, Mills SE. Squamous cell carcinoma variants of the upper aerodigestive tract. American Journal of Clinical Pathology. 2005; 124(Suppl 1):S96-S109.

3. Su HH, Chu ST, Hou YY, et al. Spindle cell carcinoma of the oral cavity and oropharynx: factor affecting outcome. $J$ Chin Med Assoc. 2006;69(10):478-483.

4. Cardesa A, Zidar N. Spindle cell carcinoma. In: L Barnes, et al. editors. J World Health Organization of Tumors. Pathology and Genetics of Head and Neck Tumors, IARC Press, Lyon, France, 2005. pp. 127-128.

5. Silvestri SB, Carrau RL, Peel R, et al. Spindle cell carcinoma of the larynx with actinomyces chondritis of the larynx and trachea. Otolaryngol Head Neck Surg. 2006;134(2):345-347.

6. Gupta R, Singh S, Hedau S, et al. Spindle cell carcinoma of head and neck: an immunohistochemical and molecular approach to its pathogenesis. $J$ Clin Pathol. 2007;60(5):472-475.

7. Katase N, Tamamura R, Gunduz M, et al. A spindle cell carcinoma presenting with osseous metaplasia in the gingiva: a case report with immunohistochemical analysis. Head Face Med. 2008;4:28.

8. Biradar MV, Dantkale SS, Abhange RS, et al. Spindle cell carcinoma of the tongue: a rare variant of squamous cell Carcinoma. Ecancermedicalscience. $2014 ; 8: 447$.

9. Samdhani S, Choudhary A, Mahanta VR, et al. Spindle cell sarcoma of larynx. Indian J Otolaryngol Head Neck Surg. 2006;58(3):305-306. 